\title{
Du donné aux données : analyse épistémologique et méthodologique de quelques effets de l'usage de données vidéographiques sur l'activité de recherche
}

From the "given" to data: epistemological and methodological analysis of some

effects of the use of video data on research

\section{Bernard Sarrazy}

\section{OpenEdition}

Journals

Édition électronique

URL : http://journals.openedition.org/educationdidactique/1556

DOI : 10.4000/educationdidactique.1556

ISSN : 2111-4838

\section{Éditeur}

Presses universitaires de Rennes

\section{Édition imprimée}

Date de publication : 30 novembre 2012

Pagination : 179-184

ISBN : 978-2-7535-2254-1

ISSN : 1956-3485

Référence électronique

Bernard Sarrazy, «Du donné aux données : analyse épistémologique et méthodologique de quelques effets de l'usage de données vidéographiques sur l'activité de recherche », Éducation et didactique [En ligne], 6-3 | novembre 2012, mis en ligne le 30 novembre 2014, consulté le 08 décembre 2020. URL http://journals.openedition.org/educationdidactique/1556 ; DOI : https://doi.org/10.4000/ educationdidactique.1556 


\title{
DU DONNÉ AUX DONNÉES : ANALYSE ÉPISTÉMOLOGIQUE ET MÉTHODOLOGIQUE DE QUELQUES EFFETS DE L'USAGE DE DONNÉES VIDÉOGRAPHIQUES SUR L'ACTIVITÉ DE RECHERCHE
}

\author{
Bernard Sarrazy (LACES, Université Bordeaux Segalen)
}

\begin{abstract}
Résumé: à partir des cinq productions scientifiques réalisées en prenant appui sur une même séquence vidéo, l'auteur analyse quelques effets méthodologiques et épistémologiques conséquents à cette situation. Comment les chercheurs se sont-ils accommodés de la non correspondance entre leur cadre théorique (habituel) et le donné dont il disposait? Lauteur conclue en proposant 'et en opposant) deux sensibilités épistémologiques à l'œuvre dans les régimes de recherche adoptés en référence aux deux déclinaisons de la vérité (aléthéia et omoisis) tel que Heidegger les a discutés.
\end{abstract}

Mots clés: Épistémologie de la recherche, méthodologie, théorie des situations didactiques, corpus vidéo.

Bernard Sarrazy

\section{Introduction}

Le but de cet article est d'analyser, à partir des cinq productions présentées dans ce numéro, les effets, principalement méthodologiques et épistémologiques, liés aux conditions d'une situation de recherche à partir d'un enregistrement vidéographique d'une séquence d'enseignement motivé initialement par un ensemble de questions d'une recherche autre. Autrement dit, il ne s'agira pas d'examiner les intérêts de ces productions prises une à une, mais bien d'identifier et analyser les effets attachés à cette situation de production. L'intérêt de cette analyse déborde, selon moi, largement le seul examen de ces effets dans la mesure où elle permet d'anticiper quelques phénomènes relatifs aux modes d'analyses des corpus vidéographiques propres au programme Visa.

Deux écueils devaient impérativement être évités: le premier est celui d'une inter-critique des recherches présentées et des méthodes utilisées pour les hiérarchiser sur une échelle de pertinence; le second est celui de la naturalisation de la situation dans laquelle les cinq chercheurs étaient placés: produire des connaissances sur un matériau qui a été conçu à l'origine pour répondre à d'autres questions que celles qu'ils ont été conduits à se poser; souvenons-nous de cette belle leçon de Bachelard dans La formation de l'esprit scientifique (1975) : «Pour un esprit scientifique, toute connaissance est une réponse à une question. S'il n'y a pas eu de question, il ne peut y avoir connaissance scientifique. Rien ne va de soi, rien n'est donné, tout est construit. ». Quelles modalités de questionnements vont émerger de cette situation? Quelles données vont émerger de ce corpus? Quels problèmes méthodologiques et épistémologiques vont se poser?

\section{Une situation « trouée »}

Commençons par rappeler que tout travail de recherche commence par une centration du regard $\mathrm{du}$ chercheur sur une contingence (construite et non donnée), susceptible de répondre aux questions initiales; cette contingence, partiellement construite par la posture théorique, et interrogée selon une focale théorique déterminée (pouvant aller du macro au micro), est censée faire exister les interrogations du chercheur, éventuellement d'y apporter des réponses et le plus souvent de faire émerger d'autres questionnements; elle est loin d'épuiser la complexité (pour ne dire la « réalité ») des systèmes et des dispositifs qui sont mis à l'étude. En effet, la contingence du chercheur s'élabore grâce aux concepts qui délimitent tout à la fois les espaces et les moyens d'observations, et qui conduisent à construire les catégories par lesquelles le « réel » sera saisi ( les bons élèves », " les classes favorisées », «les jeunes ", « les interactions », « le didactique ", le « contrat didactique »...), mais ce découpagemontage ou encore ce « jeu de l'isolation », comme le désigne Stengers (1987) ${ }^{1}$, est un jeu dangereux car il risque de " "faire taire" cela même qu'on interroge. » 


\section{DU DONNÉ AUX DONNÉES...}

(id.) ; par exemple, si les frottements furent négligés par Galilée dans ses tentatives de modélisation des pierres qui tombent, ces mêmes frottements sont loin d'être négligeables pour les oiseaux qui volent ou les voitures qui roulent. C'est bien parce qu'on a pu théoriser le phénomène de la chute des corps, qu'on a pu un jour en maîtriser les effets : déterminisme n'est pas fatalisme. Ce point aveugle est nécessairement inscrit dans la contingence et il est le prix à payer dans notre effort de connaissance du réel.

Ainsi donc, nos collègues se trouvaient face à un matériau très particulier: un donné et non un corpus, autrement dit des données issues d'une contingence autre qui, comme on vient de le voir, présupposait une intention, une instrumentation, une conceptualisation a priori... bref, une construction théoricoempirique. Or, rappelons-le, la situation proposée à ces chercheurs exigeait qu'ils élaborent une question scientifique et qu'ils tentent d'y répondre; ils ont donc dû transformer ce donné initial en une contingence dans laquelle leur questionnement pouvait exister, ou bien dégager un questionnement à partir d'une contingence possible...

Quels ont été des effets de cette situation spécifique à ce mode de production (usage de vidéos) sur les stratégies d'élaboration de connaissances en regard des cadres et des méthodes retenues par chacun d'eux? Pour y répondre, nous avons adopté la posture du « didacticien anthropologue » (au sens où nous mettons en regard les conditions de production en rapport avec les productions elles-mêmes dans l'esprit de la théorie des situations didactiques, Brousseau, 1998) pour examiner les effets spécifiques de ces conditions sur les pratiques de recherche.

Commençons par caractériser cette situation: pour filer la métaphore, on pourrait la rapprocher par certains aspects de celle des personnages du film de Jamie Uys sorti en 1980, Les dieux sont tombés sur la tête ${ }^{2}$, ou à celle des cuisiniers à qui l'on demande de faire un bon repas avec le contenu contingent d'un frigidaire. Cette dernière métaphore est intéressante car elle permet de mettre en évidence une des spécificités de cette situation qui, nous le verrons, ne sera pas sans conséquence sur les processus de production scientifique: les manques ou les trous dans le donné. La question est loin d'être triviale car il est très difficile, sinon impossible, d'estimer, à partir du seul produit « terminal », si ses qualités ou ses défauts tiennent au contenu initial du frigidaire, aux talents du cuisinier ou bien encore à l'interaction entre ses propres talents et les ingrédients dont il pouvait disposer : un cuisinier fort talentueux dans la fabrication de sauces sera fort entravé par l'absence de farine ou pire, de sel. Tous ont dû donc s'accommoder (au plein sens piagétien), tant bien que mal, de ces trous. Nous rappellerons que les débats qui ont suivi les présentations de ces recherches, ou plus largement les critiques qui sont adressées à un chercheur ou aux doctorants lors de leur soutenance de thèse... ne portent que très rarement sur les seuls résultats (des résultats isolés de leur contexte de production), ni sur les seules conditions d'observation, mais bien sur leurs interactions réciproques afin d'éprouver la validité, la consistance et donc la pertinence des premiers en regard des conditions qui ont permis de les produire. C'est dans cette perspective que nous avons analysé, au-delà même des cadres théoriques convoqués, les procédures de productions des cinq chercheurs.

\section{Perturbation et accommodation méthodologique}

Trois formes d'accommodation à cette perturbation sont apparues:

Une première consiste à faire autre chose que ce que les chercheurs auraient fait spontanément; autrement dit, dans cette première perspective, il s'agit de transformer la question initiale et qui pourtant les intéressait, pour en poser une autre dont ils pensaient pouvoir obtenir la réponse à partir de ce dont ils pouvaient disposer. Pour le dire autrement, les contraintes syntaxiques l'emportent sur les visées sémantiques - le phénomène est classique chez les « apprentis chercheurs », qui choisissent ou transforment souvent leur sujet en fonction de leur degré d'affinité avec la méthodologie qu'il nécessite (entretiens ou questionnaires le plus souvent).

D'autres ont maintenu leur projet malgré les trous dans le matériau et, plus ou moins implicitement, ont tenu pour acquis que certains indices pouvaient être pertinents pour rendre compte de ce qu'ils voulaient voir mais qu'ils ne pouvaient pas observer (ce fut le cas pour 2 ou 3 collèguesà propos des indicateurs 
qu'ils ont retenus pour dire quelques mots sur les effets d'apprentissage de la situation enregistrée) : pour apprendre, les élèves doivent nécessairement savoir cela, et cela ils peuvent l'apprendre grâce à cela (la granularité des savoirs, les formes répétitives, etc.), mais rien ne permet de savoir in fine si ces indices sont valides et empiriquement robustes; dans ce cas, l'affirmation de cette validité de ces indices sur ce mode est davantage un postulat qu'un énoncé logico-empirique (comme dirait Piaget). En d'autres termes, la pertinence a priori pour nécessaire qu'elle soit, ne suffit pas à fonder la validité et la robustesse du cadre.

D'autres enfin, comme Laurent Talbot, n'ont pas transformé leur questionnement initial et leur intérêt scientifique, mais ont modifié la situation proposée en comblant les manques que leur projet nécessitait en allant rechercher des données qui n'étaient pas présentes.

Bref, trois formes d'accommodation aux particularités de cette situation peuvent être ici identifiées:

1. celle du refoulement ou de l'évitement ( « je vais faire autre chose »);

2. celle de la compensation partielle par la production d'implicite ou la mise en avant de postulats hypothétiques (« je vais faire comme si »);

3. celle enfin de la transgression à visée compensatoire consistant à combler les lacunes pour engager et traiter le projet initial.

\section{Perturbation et sensibilité épistémologique}

Rappelons-le, il s'agissait pour nos collègues d'examiner et d'analyser cette situation pour dire quelque chose sur les rapports complexes entre l'action du professeur (enseigner) et l'apprentissage des élèves. Or, le matériau ne fournissait aucun observable permettant de répondre à cette délicate question de savoir si les élèves avaient appris: que savent-ils faire, dire, expliquer... qu'ils ne savaient pas faire avant? Quelles questions peuvent-ils désormais poser qu'ils ne pouvaient pas précédemment poser? Quels obstacles ont-ils dépassés? Ces effets sont-ils homogènes sur l'ensemble des élèves? etc.
Pour palier ce manque, deux postures épistémologiques sont apparues dans les présentations que l'on pourrait caractériser à partir des deux conceptions de la vérité telles que Heidegger les a théorisées: aléthéia et omoisis.

Aléthéia, mot grec constitué du radical léthé signifiant l'oubli et du préfixe privatif « $\mathrm{a}$ » signifie littéralement: « un discours qui s'oppose à l'oubli ». La vérité est un discours de révélation qui vise à lever le voile sur ce qui est masqué ou caché: «À l'origine vérité [aléthéia] veut dire: ce qui a été arraché à une occultation. La vérité est cet arrachement, toujours en mode de dévoilement » (Heidegge, 1968, 143).

Avec l'omoisis, l'autre type de vérité, nous changeons de régime: le discours n'est plus orienté vers la révélation comme précédemment, mais sur l'adéquation entre son contenu propositionnel et la chose: adaequatio intellectus et rei (la représentation est adéquate à la chose).

Même si l'opposition n'est pas ici aussi radicale, elle s'avère assez pertinente pour distinguer les régimes de validité à l'œuvre dans les productions qui ont été présentées:

Dans certains cas, on a pu observer une validité qui se fonde principalement sur la nécessité théorique: l'empirique est alors placé sous le joug $\mathrm{du} \operatorname{logos}, \mathrm{du}$ discours théorique dans sa fonction de dévoilement (aléthéia). Or, comme le pointe Bachelard dans la Psychanalyse du feu :

«Il suffit que nous parlions d'un objet pour nous croire objectifs. Mais par notre premier choix, l'objet nous désigne plus que nous ne le désignons et ce que nous croyons nos pensées fondamentales sur le monde sont souvent des confidences sur la jeunesse de notre esprit. [...] l'évidence première n'est pas une vérité fondamentale. En fait, l'objectivité scientifique n'est possible que si l'on a d'abord rompu avec l'objet immédiat, si l'on a refusé la séduction du premier choix, si l'on a arrêté et contredit les pensées qui naissent de la première observation. » (Bachelard, 1985, 11).

Dans d'autres cas, la validité est fondée sur la présomption d'une correspondance entre les relations intra-corpus (objectivées) avec les trous: ce fut le 


\section{DU DONNÉ AUX DONNÉES...}

\section{Bernard Sarrazy}

cas chez Andrée Tiberghien, Marie-Pierre Chopin et Abdelkarim Zaid qui ont établi par exemple des relations intra-temporelles et des relations entre thèmes.

Il est d'ailleurs intéressant de noter que les questions qui ont été posées portaient sur des présentations de type plutôt aléthéia (et donc à forte dominante herméneutique) et que, dans ce cas, les réponses étaient beaucoup fermes que celles qui s'adressaient à des présentations de type omoisis dans lesquelles les réponses étaient plus nuancées, dubitatives ou hypothétiques.

On l'aura reconnu, on débouche ici sur le vieux problème poppérien de la falsifiabilité qui ne me semble pas spécifique de cette situation de recherche (usage de la vidéo) mais qui paraît amplifié par rapport aux situations ordinaires de recherche:

- Andrée Tiberghien clôture d'ailleurs son exposé sur ce thème en initiant une discussion sur la distance entre observables et interprétation;

- Laurent Talbot tente par l'entretien qu'il réalise avec le professeur de valider le résultat de ses observations;

- Laurent Lima regrette de ne pas pouvoir appuyer son hypothèse de l'erreur liée à la cooccurrence des termes température / raies par une observation de ce qu'ont appris (ou non) les élèves;

- Marie-Pierre Chopin tente de montrer la cohérence de sa recherche et la consistance de ses résultats par la mise en correspondance de son intuition inspirée par ses cadres théoriques et l'objectivation à laquelle elle se livre;

- enfin Abdelkarim Zaid annonce un programme plus heuristique et réflexif à partir des observations disponibles, mais éloigné de ses intérêts scientifiques habituels.

\section{Conclusion}

Toute recherche vise la production de connaissances nouvelles, et le caractère de nouveauté se manifeste par les effets de sens qu'elles produisent par les relations qu'elles établissent entre des dimen- sions a priori non liées, et qui présentent des intérêts définis en terme d'explication, de compréhension, à tout le moins de clarification par la mise à jour des conditions (méthodologiques) et des principes (épistémologiques) qui ont présidé à leur production. Ainsi, la construction de ces connaissances ne se réduit ni à la seule dimension empirique du matériau, ni à la seule dimension théorique (idéaliste), mais bien dans la nature (fragile et délicate) des rapports de ces deux dimensions. Ces cinq recherches se différencient assez clairement sur ce point; en effet, comme on a pu le voir, le sens se tisse soit à l'interne (entre des observables intra-corpus), soit à l'externe (des observables intra-corpus sont mis en lien avec des « dimensions », parfois supposées, extra-corpus). Enfin, on notera que ces processus de tissage s'avèrent assez bien ajustés aux positions des chercheurs sur l'axe théorie-empirie (dominante plutôt empirique: perspective « omoiosis »vs plutôt théorique, idéaliste avec la perspective « aléthéia »). Ces modes d'ajustement peuvent se comprendre à partir des rapports qui se sont établis entre les cadres (théoriques) et les contenus (empiriques) dans cette situation trouée. Si nous considérons le cadre à la manière de Wittgenstein (1961) comme un filet apposé sur le réel selon sa structure, la forme et la taille de ces mailles, le chercheur va recueillir des données fort différentes dans leur nombre, leur degré de granularité, bref dans leur qualité ; mais remarquons bien que cette qualité n'est pas intrinsèque aux données elles-mêmes, mais tient au caractère spécifique des mailles du filet dans son rapport au projet scientifique qui l'a fait naître et qui a permis de les produire. Le contenu est donc nécessairement déterminé par le cadre, mais pas entièrement - dans ce cas, on assisterait au meurtre parfait du réel, une sorte d'idéalisme radical en quelque sorte.

Ce problème de l'ajustement des cadres et des contenus n'est pas nouveau dans le champ des théories de la connaissance, Piaget l'avait clairement abordé dans Biologie et connaissance, et sa position constructiviste permettait d'éviter le double écueil d'un empirisme naif et d'un idéalisme béat:

« La solution la plus simple pour expliquer l'accord de ces cadres et de leurs contenus consiste naturellement à admettre que les contenus agissent en retour sur les cadres et qu'ainsi l'adaptation à expliquer s'effectue par tâtonnements progressifs, autrement dit par le moyen 
d'une équilibration entre assimilation des contenus aux cadres et l'accommodation différenciatrices des cadres aux contenus. » (Piaget, 1967, 473)

Autrement dit, c'est bien dans le processus de tissage des liens, que j'évoquais plus haut, que s'opère cet ajustement. Telle est selon moi, la plus importante difficulté que nos collègues ont rencontrée dans cette situation. Certains ont ignoré les trous, d'autres les ont comblés par les cadres, d'autres enfin ont produit des conjectures (raisonnables mais pas nécessairement avérées) sur la nature des trous. En d'autres termes nos collègues, bien qu'ayant le même support, n'avaient pas les mêmes données, et leurs filets étaient plus ou moins adéquats aux potentialités des données à leur disposition. La richesse méthodologique de leur différence, la variété des connaissances produites, la finesse avec laquelle ils ont "rebouché » ou " évité » les trous, ne doit pas nous faire oublier les écueils que j'ai tenté de pointer à propos de la tendance propre à cette situation trouée, d'une herméneutique radicale, ou d'un empirisme naif. Sur ce dernier point nous rejoindrons A. Marchive qui, s'appuyant tout la fois sur Guy Brousseau (1996) et Claude Levi-Strauss, soulignait l'importance, pour ne pas dire la nécessité, de l'imbrication étroite de l'aléthéia et de l'omoiosis dans l'activité scientifique, tout en prenant garde de bien les distinguer: "L'observation des faits et de l'élaboration des méthodes permettant de les utiliser pour construire des modèles ne se confondent jamais avec l'expérimentation au moyen des modèles euxmêmes " (Lévi-Strauss, 1985, 333). Seule, selon nous, la vigilance épistémologique, au sein de nos communautés scientifiques, constitue un garde-fou contre ces deux dérives en même temps qu'une disposition puissante permettant de les conjuguer. Telle est, selon moi, une responsabilité majeure de notre travail de chercheur. 


\section{DU DONNÉ AUX DONNÉES...}

\section{NOTES}

1. «L'isolation, nous dit I. Stengers, est un jeu dangereux, et celui qui croit purifier son objet intervient en fait activement dans la signification de ce qu'il observe. [...] Chaque question est un pari à propos de ce à quoi l'objet interrogé est sensible et aucune méthode n'est neutre par rapport à ce problème. » $(1987,349)$.

2. Voici le synopsis (consultable sur wikipedia. org, nov. 2012); « Dans une tribu isolée de Bochimans du Kalahari, sans contact avec la 'civilisation', se produit un miracle: un objet extraordinaire est tombé du ciel. Transparent et très dur, il peut servir de pilon, de flûte, de récipient et de bien d'autres choses encore. Un cadeau des dieux pour ce peuple sympathique où l'on partage tout. Oui mais... Il s'agit en fait d'une vulgaire bouteille de Coca-Cola, qu'un aviateur peu scrupuleux a jetée par-dessus bord. Cette bouteille est si utile que tout le monde en a besoin en même temps. La belle entente d'autrefois fait place à des querelles incessantes. Pour avoir fait un tel cadeau, pas de doute, les dieux sont tombés sur la tête. Le conseil se réunit et décide que Xhixho ira au bout du monde pour rendre aux dieux leur cadeau empoisonné. »

\section{RÉFÉRENCES}

Bachelard G. (1975). La formation de l'esprit scientifique: contribution à une psychanalyse de la connaissance objective. Paris: Vrin, 257 p.

Bachelard G. (1985). La psychanalyse du feu, éd. Gallimard, Folio essais.

Brousseau G. (1996). L'enseignant dans la théorie des situations didactiques. Actes de la VIII École et Université d'Été de didactique des mathématiques. IREM Clermont-Ferrand, 1996, p. 3-46.

Brousseau G. (1998). Théorie des situations didactiques, [Textes rassemblés et préparés par N. Balacheff, M. Cooper, R. Sutherland, V. Warfield], Grenoble: La pensée sauvage. 395 p., coll. Recherches en didactique des mathématiques.

Heidegger M. (1968). Questions II. Paris: Gallimard, 276 p.

Levi-Strauss C. (1971). Mythologiques IV. L'homme nu. Paris: Plon, 688 p.

Marchive A. (2008). La pédagogie à l'épreuve de la didactique: Approche historique, perspectives théoriques et recherches empiriques. Rennes: PUR. 152 p.

Piaget J. (1967). Biologie et connaissance: essai sur les relations entre les régulations organiques et les processus cognitifs. Paris: Gallimard, 430 p.

Stengers I. (dir) (1987). D'une science à l'autre: des concepts nomades. Paris: Le Seuil. 387 p., coll. Science ouverte.

Wittgenstein L. (1961). Tractatus logico-philosophicus suivi des Investigations philosophiques. Paris: Gallimard. $364 \mathrm{p}$. 